\title{
Mechanochemical Prebiotic Peptide Bond Formation
}

\author{
Tomislav Stolar, ${ }^{1}$ Saša Grubešić, ${ }^{2}$ Nikola Cindro, ${ }^{3}$ Ernest Meštrović, ${ }^{2}$ Krunoslav Užarević*, ${ }^{1}$ \\ and José G. Hernández* \\ ${ }^{1}$ Ruđer Bošković Institute, Bijenička c. 54, 10000 Zagreb, Croatia \\ ${ }^{2}$ Xellia Pharmaceuticals, Slavonska avenija 24/6, 10000 Zagreb, Croatia \\ ${ }^{3}$ Faculty of Science, University of Zagreb, Horvatovac 102a, 10000 Zagreb, Croatia
}

\begin{abstract}
The presence of amino acids on the prebiotic Earth, either stemming from endogenous chemical routes or delivered by meteorites, is consensually accepted. In contrast, prebiotically plausible pathways to achieve peptides from unactivated amino acids are still unclear since most oligomerization approaches rely on thermodynamically disfavored reactions in solution.
\end{abstract} Alternative hypotheses such as the prebiotic impact scenario postulate that the mechanical impacts from meteorites and geochemical phenomena played an important role in delivering exogenous material to Earth, thus providing the geochemical, mechanical, and thermal conditions to synthesize small prebiotic organic compounds in the absence of bulk liquid media. In this context, here we evaluate the applicability of mechanochemistry by ball milling for peptide bond formation under a prebiotic impact scenario. We found that the combination of mechanical forces and prebiotically plausible and ubiquitous minerals as activators enable the oligomerization of amino acids such as glycine in the absence of bulk water (or solvents) and at ambient temperature. Increasing the mechanochemical reactor's temperature is shown to favor the degree of polymerization concomitantly with the formation of cyclic glycine dimer [cyclo(Gly 2$)$ or DKP], a product commonly considered as a dead-end in solution peptide bond formation. However, our study shows that DKP can be mechanochemically activated and used as a source for glycine oligomers. The findings of this research provide alternative mechanochemical routes towards oligopeptides and establish new synthetic approaches for 
prebiotic chemistry that are not limited by poor diffusion of the reactants, thus complementing the current alternating wetting and drying prebiotic environment strategy.

\section{Introduction}

Peptide bond formation is considered as one of the critical chemical transformations in the field of prebiotic chemistry. ${ }^{1-3}$ On the one hand, it has been proposed that peptides could have behaved as catalysts for the formation of other prebiotically relevant building blocks in an early Earth scenario. ${ }^{4}$ Additionally, the presence of peptides in a primitive Earth has been postulated as favorable to establish an ancient molecular symbiosis with nucleic acids. ${ }^{5-8}$ Therefore, it is not surprising that once the formation of amino acids during the iconic Miller-Urey experiment was confirmed, ${ }^{9}$ various studies have attempted to synthesize peptides from amino acids under prebiotic conditions. ${ }^{10-15}$ Oligomerization of amino acids has been investigated under fluctuating environments such as alternating hot-dry/cool-wet water evaporation and rehydration cycles. ${ }^{16-19}$ One of the main challenges of this approach is the poor diffusion of the solid reactants during the hot-dry period, limiting the elongation of peptides. ${ }^{20}$ Therefore, establishing a new synthetic approach that is not limited by poor diffusion of the reactants under dry conditions could significantly improve the current alternating wetting/drying prebiotic strategy, for example, by overcoming the need for wet cycles. In the search for potential prebiotic conditions to carry out chemical reactions under dry conditions, the prebiotic impact scenario appears as a promising alternative. It is well documented that Earth underwent a meteoritic bombardment that caused unmistakable changes to our planet. ${ }^{21}$ Also, the landing of meteorites on Earth could have facilitated the delivery of exogenous material as demonstrated after the detection of amino acids, ${ }^{22,23}$ dipeptides, and protein-like structures in meteorites. ${ }^{24,25}$ Moreover, the mechanical impacts from meteorites or terrestrial lithospheric activity (Figure 1a-b) could have provided the geochemical, mechanical, and thermal energy to drive chemical reactions in the absence of bulk liquid media. Although high-energy-density events could lead 
to unproductive bond cleavage in a growing peptide chain, moderate forces may benefit chain elongation. ${ }^{26}$

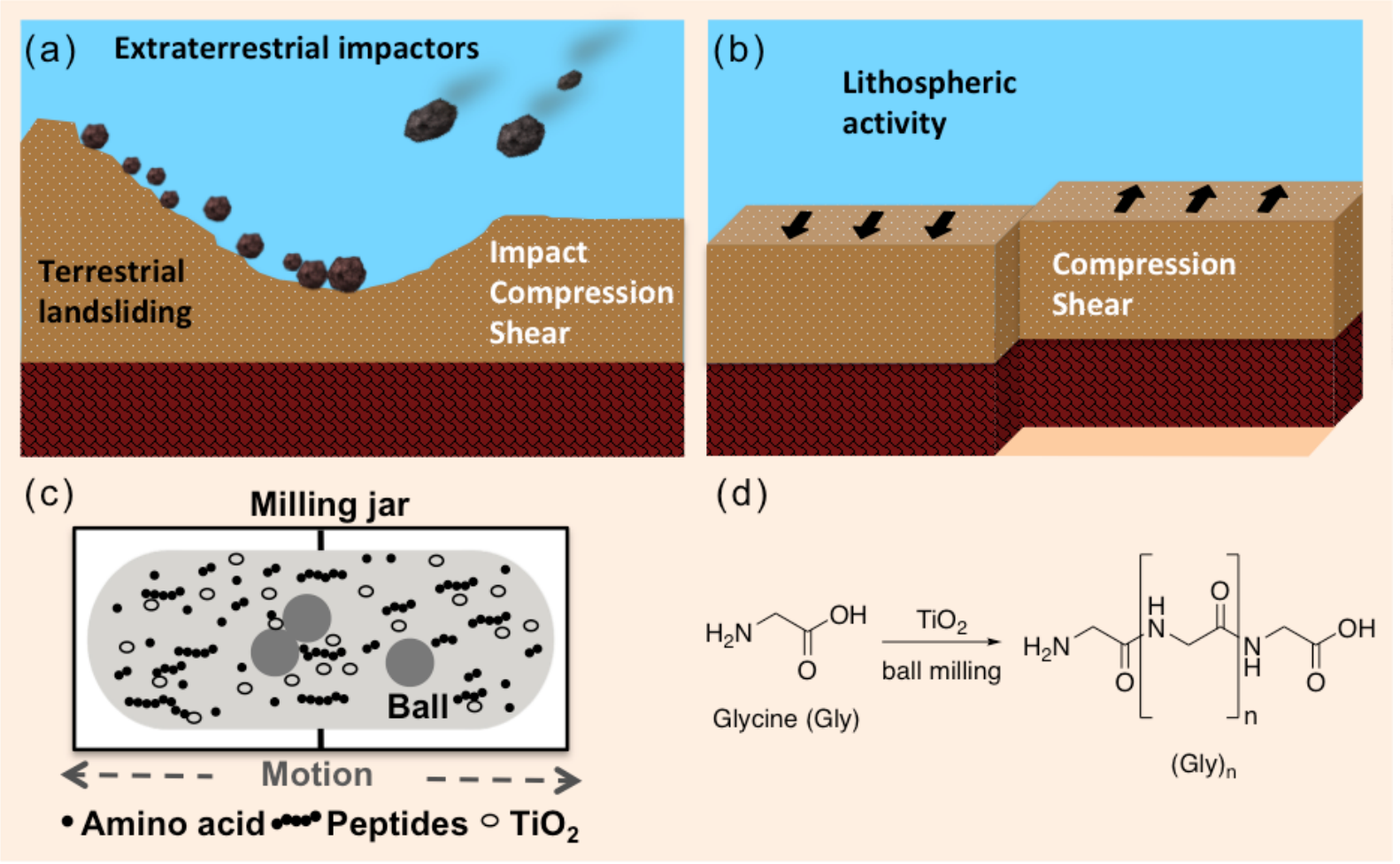

Figure 1. Prebiotic scenarios that could have provided mechanical energy by impact, compression, and shear forces: (a) extraterrestrial and terrestrial collisions and (b) plate tectonic movement. (c) Ball mill containing reactants: amino acids, peptides, and $\mathrm{TiO}_{2}$; (d) solvent-free mechanochemical peptide bond formation by ball milling.

In synthetic chemistry, one of the most efficient ways to carry out chemical transformations in the absence of a liquid media involves implementing mechanochemical techniques such as ball milling. ${ }^{27-29}$ Inside ball milling reactors, substances are subjected to compression and shear forces promoted by milling balls. The intimate mixing achieved by ball milling not only surpasses diffusion constraints when reacting bulk solids, but it can also induce new chemical reactions in the absence of solvents. ${ }^{30}$ Although the reaction conditions inside a milling reactor may seem hostile, amino acids, peptides, and enzymes have been reported to withstand the 
mechanical stress under ball milling conditions. ${ }^{31-35}$ Moreover, mechanochemistry has proven suitable for synthesizing prebiotically relevant building blocks such as $\alpha$-aminonitriles, ${ }^{36,37}$ monosaccharides, ${ }^{38,39}$ and nucleotides. ${ }^{40,41}$ Solid-state approaches have led to self-assembly of model nucleobases as well. ${ }^{42}$ Altogether, these studies have provided direct evidence of the potential of mechanical energy to drive prebiotically relevant transformations, especially in the context of a prebiotic landscape.

Here we present the study of controllable mechanical activation (Figure 1c) as a prebiotically plausible route to peptide bond formation (Figure 1d) and oligomerization of the unactivated proteinogenic amino acid, glycine (Gly), in the absence of water or other bulk solvents. We have established here that the mechanochemical oligomerization by ball milling is achieved even at ambient temperatures. However, the addition of minerals such as $\mathrm{TiO}_{2}$ is critical for the process. The reaction products were analyzed primarily by the modified ion-pair highperformance liquid chromatography (IP-HPLC) method, ${ }^{43}$ and by ultra high-performance liquid chromatography coupled with mass spectrometry (UPLC-MS). The mechanochemical reactions performed in a thermally-controlled milling reactor revealed that the degree of oligomerization increases with the temperature to the point when the reaction is directed towards forming 2,5-diketopiperazine (DKP), often regarded as an "unwanted" product that diminishes the availability of free amino acids for the subsequent oligomerization reactions. ${ }^{2,44}$ Moreover, the presence of added water in liquid-assisted grinding (LAG) experiments did not significantly inhibit the oligomerization reaction. We have also studied the mechanochemical peptide bond formation starting from Gly derivatives such as DKP, Gly2, and Gly 3 , and have established that the mechanochemical oligomerization is a dynamic process involving simultaneous formation and hydrolysis of the peptide bonds. The temperature-controlled mechanochemical processing was also attempted for the oligomerization of L-alanine. 


\section{Results and discussion}

As an initial experiment, we milled pristine glycine (Gly) at room temperature (RT) and subsequently analyzed the reaction mixture applying the modified IP-HPLC method developed by the Bracher group using a UV-Vis detector set to record the absorbance at $195 \mathrm{~nm}$ (for

details, see SI). ${ }^{43}$ The chromatographic analysis of the white powder collected after the mechanochemical treatment revealed the formation of DKP and traces of $\mathrm{Gly}_{2}$ and $\mathrm{Gly}_{3}$ (Figure 2a). This was confirmed after comparison with pure standards (Figure 2c-f). 


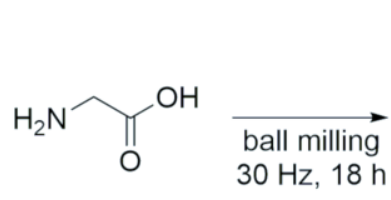

Gly<smiles>CCC(=O)NCC(=O)NCC(=O)O</smiles>

Gly<smiles>O=C1CNC(=O)CN1</smiles>

DKP

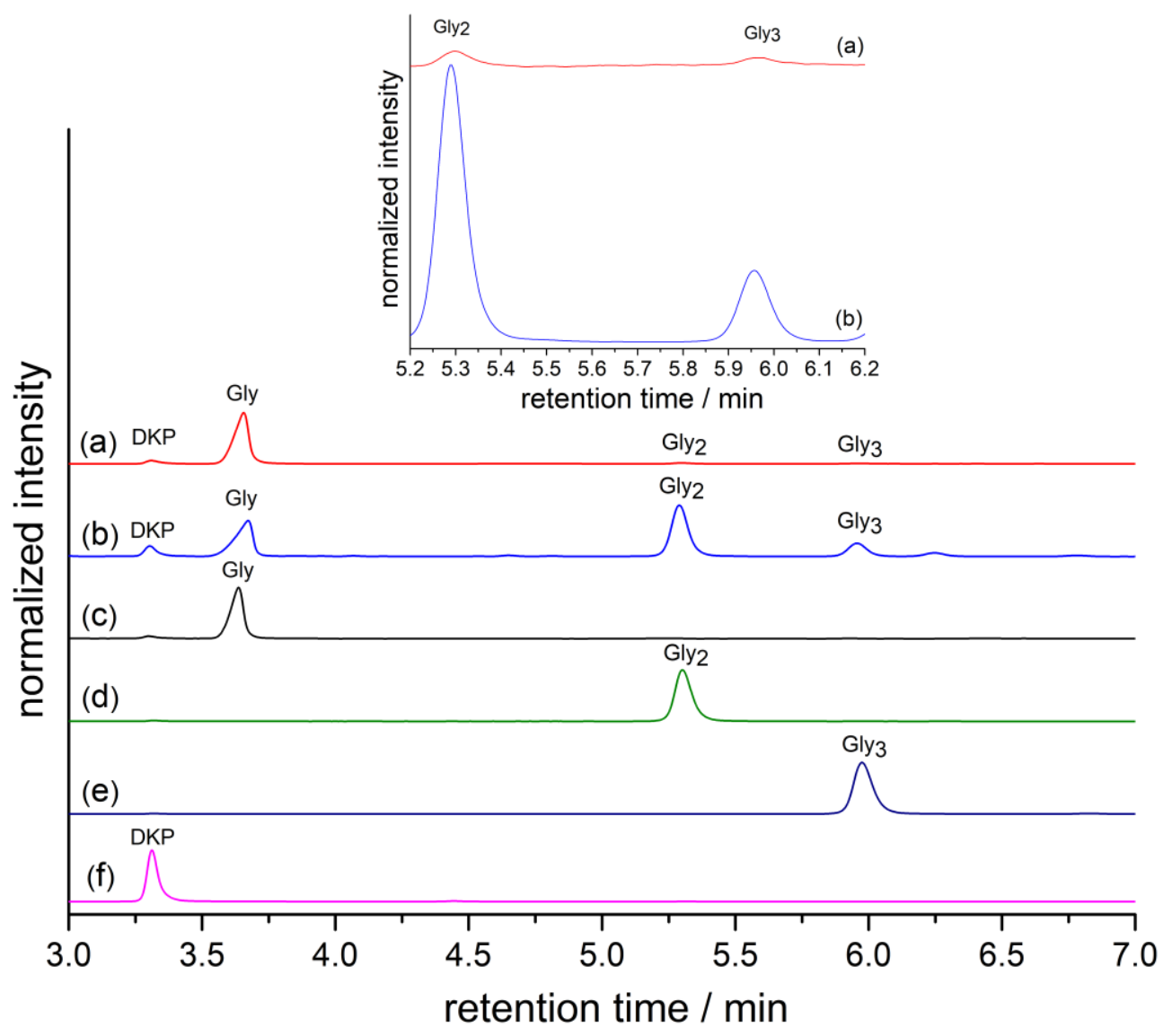

Figure 2. IP-HPLC chromatograms for (a) pristine Gly and (b) Gly and $\mathrm{TiO}_{2}$ mixture milled at RT; (c) Gly standard; (d) Gly2 standard; (e) Gly 3 standard; (f) DKP standard. The inset shows the zoomed region and reveals the presence of Gly 2 and Gly 3 in (a) and (b).

Additionally, to study the effect of the temperature on the peptide bond formation, we applied a recently described temperature-controlled ball milling protocol ${ }^{45}$ For example, in a standard experiment, Gly (60 mg, $0.80 \mathrm{mmol}$ ) was milled with a five-fold molar excess of $\mathrm{TiO}_{2}$ (anatase), at $30 \mathrm{~Hz}$ for $18 \mathrm{~h}$ and temperatures ranging from room temperature to $130{ }^{\circ} \mathrm{C}$, thus respecting practical thermal limits for prebiotic peptide bond formation, ${ }^{18,19}$ while acknowledging the expected dilution of amino acids on mineral surfaces under prebiotic conditions (Figure 1d). 
The efficiency of the mechanochemical oligomerization of glycine and the formation of higher oligomers increase at higher milling temperatures (Figure 3 and Table 1). On the one hand, raising the milling temperature gradually increases the total yield of Gly converted to linear oligomers $\left(\mathrm{Gly}_{\geq 2}\right)$ (Table 1; for quantification details, see SI). The maximum total yield is calculated to be $10.2 \%$ for milling at $100{ }^{\circ} \mathrm{C}$, as it seems to be the most optimum milling temperature (Figure 3d). On the other hand, despite having detected the longest oligomer $\left(\mathrm{Gly}_{10}\right)$ after milling at $130{ }^{\circ} \mathrm{C}$, the analysis by IP-HPLC revealed that DKP was the major component of the product mixture (Figure 3e).

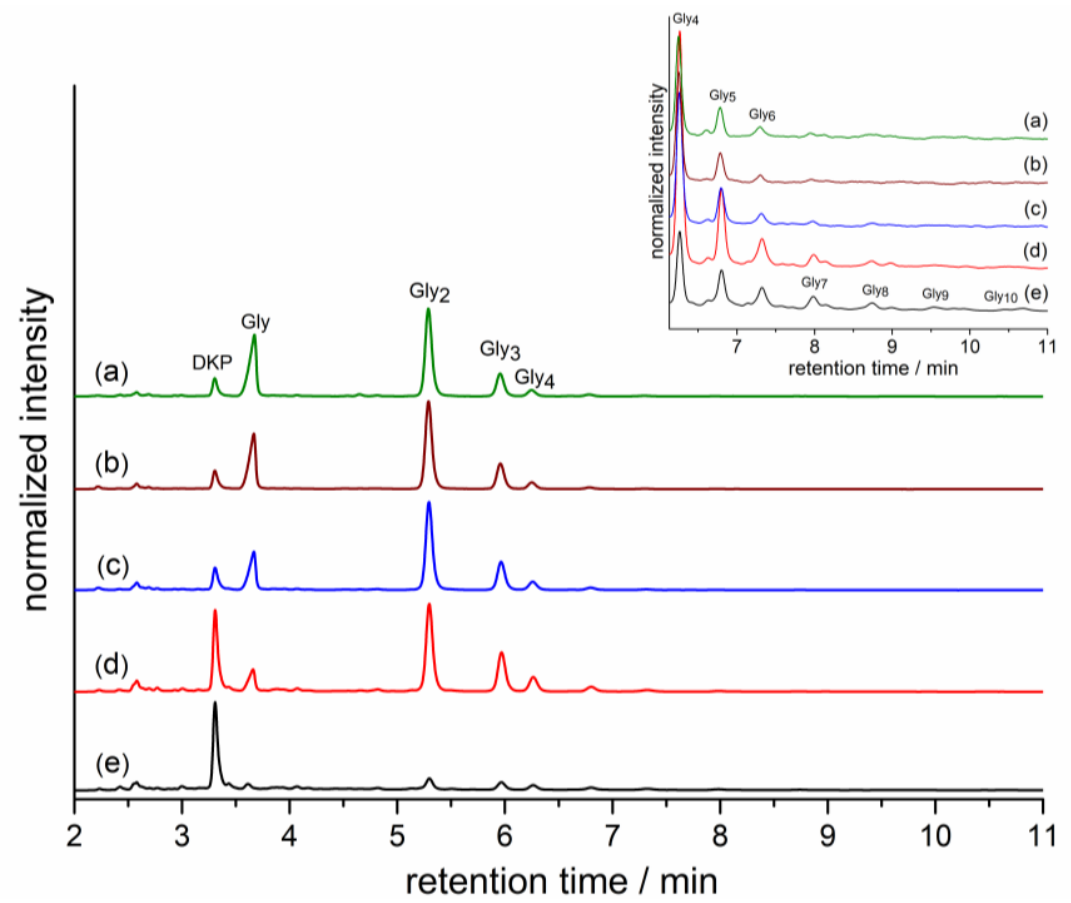

Figure 3. IP-HPLC chromatograms for Gly milled with $\mathrm{TiO}_{2}$ at (a) $\mathrm{RT}$, (b) $40{ }^{\circ} \mathrm{C}$, (c) $70{ }^{\circ} \mathrm{C}$, (d) $100{ }^{\circ} \mathrm{C}$, (e) $130{ }^{\circ} \mathrm{C}$. The inset shows the zoomed region of retention times in the $6.1-11 \mathrm{~min}$ range. 
Table 1. Effect of the milling temperature on the mechanochemical oligomerization of Gly with $\mathrm{TiO}_{2}$.

\begin{tabular}{|c|c|c|}
\hline Milling temperature $\left({ }^{\circ} \mathrm{C}\right)$ & Longest detected oligomer $^{[a]}$ & Yield $(\%)^{[\mathrm{b}]}$ \\
\hline RT & Gly $_{6}$ & 6.7 \\
\hline 40 & $\mathrm{Gly}_{6}$ & 6.7 \\
\hline 70 & $\mathrm{Gly}_{7}[\mathrm{cc}]$ & 10.1 \\
\hline 100 & $\mathrm{Gly}_{8}$ & 10.2 \\
\hline 130 & $\mathrm{Gly}_{10}[\mathrm{~d}]$ & 8.5 \\
\hline
\end{tabular}

(a) Based on IP-HPLC analysis using a UV-Vis detector set to record the absorbance at $195 \mathrm{~nm}$. (b) The combined yield of all of the linear oligomers of glycine Gly $\mathrm{y}_{\geq 2}$. (c) The presence of Gly 8 was detected by the UPLC-MS method (Figure S12). (d) The presence of Gly ${ }_{11}$ was detected by UPLC-MS (Figure 4).

To unambiguously determine the presence of all of the oligomers of glycine and to corroborate the degree of oligomerization, we developed an analytical method based on UPLC-MS (for details, see SI). Analysis of the samples from previous experiments using a Q-TOF mass detector confirmed the presence of linear oligomers of glycine. Importantly, in some cases, the analysis of the milled samples by UPLC-MS revealed the presence of longer Gly $\mathrm{y}_{\mathrm{n}}$ compared with the preliminary analysis by IP-HPLC. For example, the solid products obtained after milling Gly at $70{ }^{\circ} \mathrm{C}$ and $130{ }^{\circ} \mathrm{C}$ were found to contain Gly8 and Gly 11 , respectively (Figure S12 and Figure 4). 


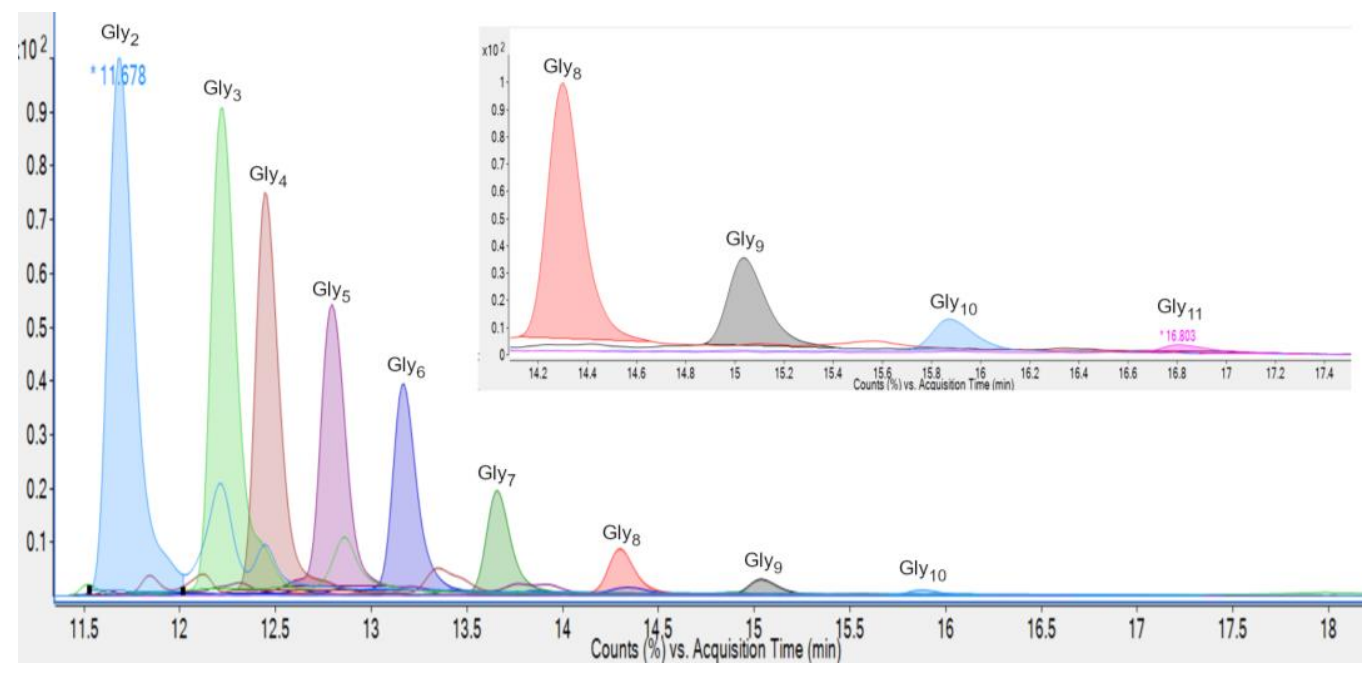

Figure 4. UPLC-MS analysis of Gly milled with $\mathrm{TiO}_{2}$ at $130{ }^{\circ} \mathrm{C}$. The inset shows the zoomed region in the acquisition time range from 14-17 min. Note: due to differences in the sample preparation $(\mathrm{pH})$ the retention times of the oligomers by UPLC-MS differ from the ones found by IP-HPLC analysis.

At this point in the research, we had demonstrated that under ball milling conditions, mixtures of Gly and $\mathrm{TiO}_{2}$ underwent oligomerization. However, to better understand the dynamics and mechanism of the mechanochemical peptide bond formation, we performed additional peptide bond formation experiments in the presence of $\mathrm{TiO}_{2}$ starting from $\mathrm{Gly}_{2}$ and $\mathrm{Gly}_{3}$ instead of Gly. After $18 \mathrm{~h}$ of milling at $130{ }^{\circ} \mathrm{C}$, the reaction of $\mathrm{Gly}_{2}$ and $\mathrm{Gly}_{3}$ with $\mathrm{TiO}_{2}$ afforded once again a mixture of oligopeptides (Figure 5, Figures S13 and S14). The oligomerization of Gly afforded $^{2}$ the longest oligopeptide obtained by milling at $130{ }^{\circ} \mathrm{C}$, Gly 14 (Figure S9). Interestingly, oddnumber oligomers of glycine and even-number oligomers of glycine were observed when Gly2 and Gly 3 were used as starting materials, respectively, showing that the formation of the peptide bond is a dynamic and reversible process under the mechanochemical reaction conditions. Additionally, the presence of DKP in the reaction mixtures after the mechanochemical treatment of $\mathrm{Gly}_{2}$ or $\mathrm{Gly}_{3}$ (Figure 5b-c) led us to believe that DKP could be a productive intermediate in the oligomerization of Gly rather than an undesirable byproduct in prebiotic 
peptide formation studies. ${ }^{2,44}$ Along these lines, milling DKP at RT and in the presence of $\mathrm{TiO}_{2}$ was shown to generate oligomers up to Gly 10 (Figure 5d and Figure S16).

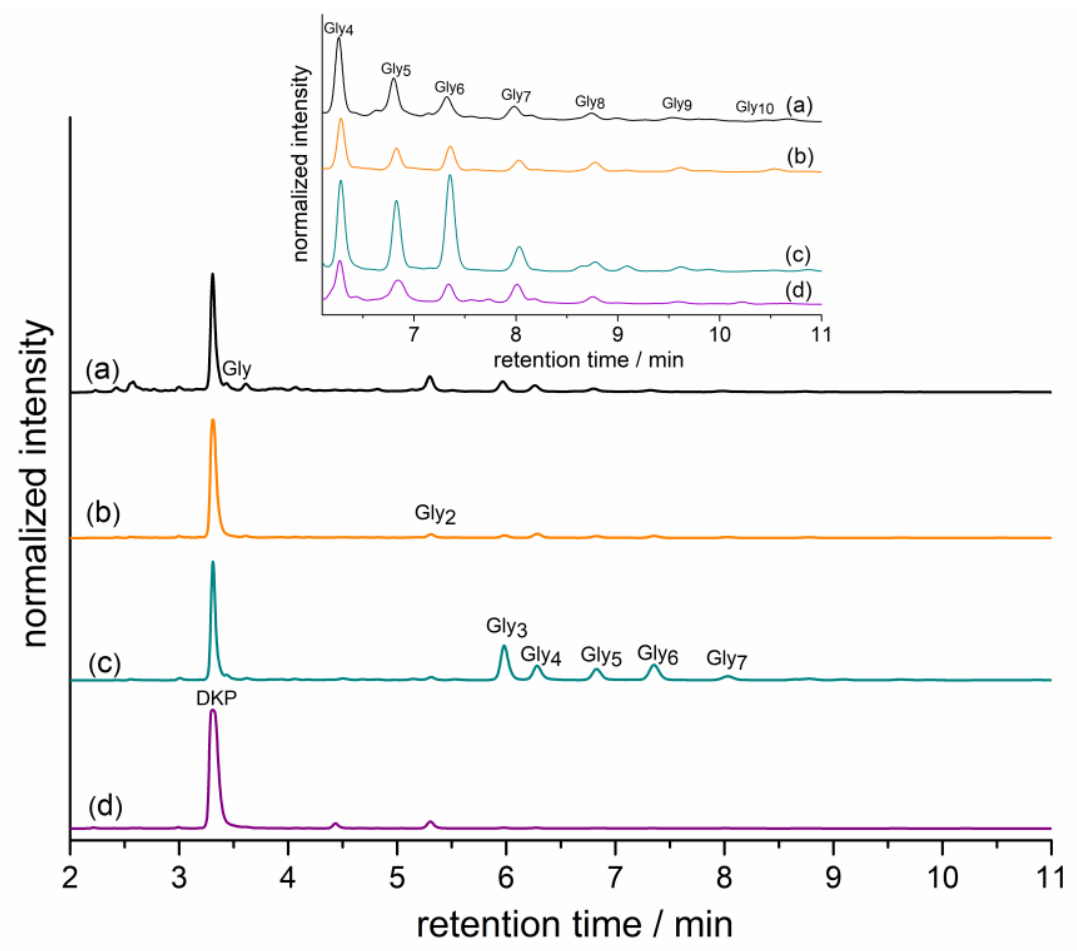

Figure 5. IP-HPLC chromatograms: (a) Gly milled with $\mathrm{TiO}_{2}$ at $\mathrm{RT}$, (b) $\mathrm{Gly}_{2}$ milled with $\mathrm{TiO}_{2}$ at $130{ }^{\circ} \mathrm{C}$, (c) Gly 3 milled with $\mathrm{TiO}_{2}$ at $130{ }^{\circ} \mathrm{C}$ and (d) DKP milled with $\mathrm{TiO}_{2}$ at $130{ }^{\circ} \mathrm{C}$. The inset shows the zoomed region of retention times in the 6.1-11 min range.

The formation of peptide bonds can also be determined by ex-situ attenuated total reflectanceFourier transform infrared (ATR-FTIR) spectroscopy (Figure 6a). Despite the broadening of the spectral bands in the milled samples, a gradual shift towards higher wavenumbers for the amide $\mathrm{C}=\mathrm{O}$ stretching region, as the temperature of the milling increased, was noticed. Based on previous reports, this observation was taken to indicate the success of the mechanochemical peptide bond formation (Figure 6a). ${ }^{46,47}$ In particular, the spectroscopic changes were most pronounced in the case of the milling reactions carried out at $130{ }^{\circ} \mathrm{C}$ (Figure 6a). To inspect the product in more details, the milled sample was dissolved in water and filtered. The organic material recovered after the evaporation of the filtrate (Figure S19) was analyzed by ATR-FTIR 
spectroscopy, together with standards of pure Gly2 and Gly 3 (Figure 6b). The broad band with the maximum at $1652 \mathrm{~cm}^{-1}$ and the weak band at around $3300 \mathrm{~cm}^{-1}$ ascribed to $\mathrm{N}-\mathrm{H}$ stretching indicate the presence of oligopeptides in the mixture. However, it is evident that the chromatographic analytical methods (IP-HPLC and UPLC-MS) were more appropriate to gain more in-depth insight into the oligomerization process.

(a)

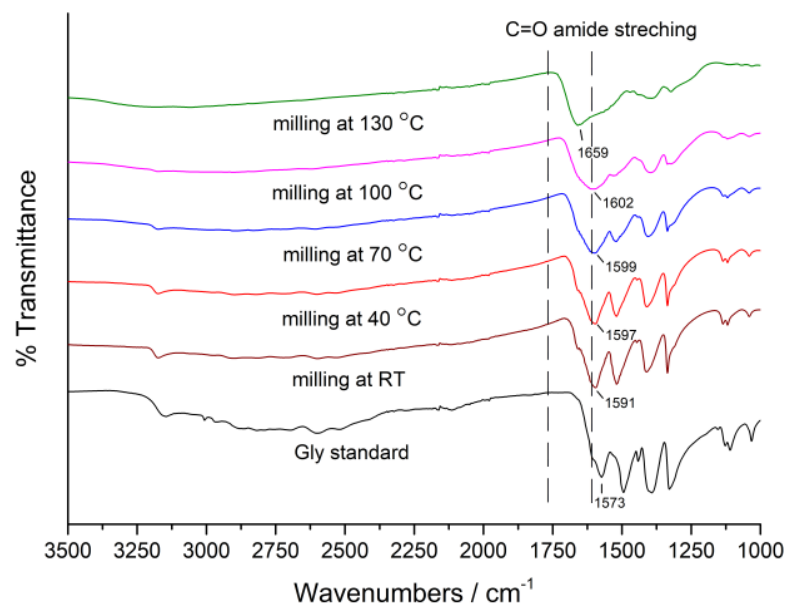

(b)

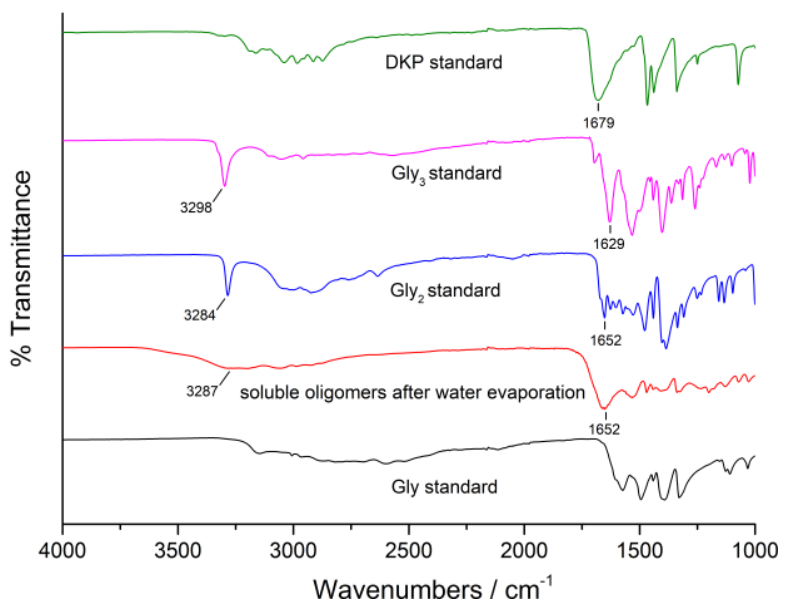

Figure 6. (a) ATR-FTIR analysis of the raw reaction mixtures after ball milling. (b) ATR-FTIR analysis of the recovered organic material after filtration of the milling experiment at $130{ }^{\circ} \mathrm{C}$ (soluble oligomers after water evaporation) and comparison with standards.

From the prebiotic plausibility standpoint of the mechanochemical peptide bond formation, it would be expected that the process would be robust and not exclusive for a single mineral surface. Hence, we attempted the formation of peptides on other ubiquitous minerals such as $\mathrm{SiO}_{2},{ }^{48}$ and sheeted montmorillonite and mica silicates. The mechanochemical Gly oligomerization was successful with all of the minerals mentioned above (Figures S22-S24). Furthermore, having performed LAG reactions with water, we show that mechanochemical peptide bond formation proceeds even in small amounts of water at $\mathrm{RT}$ and at $130{ }^{\circ} \mathrm{C}$ (Figures S25 and S26). Finally, to prove the generality of the proposed mechanochemical peptide bond formation, milling L-alanine (L-Ala) in the presence of $\mathrm{TiO}_{2}$ also results in the formation of $\mathrm{L}-$ Ala oligomers (Figures S27 and S28). 


\section{Conclusion}

The feasibility of a prebiotic impact scenario to nurture the synthesis of amino acid derivatives has recently been demonstrated. ${ }^{36}$ However, the formation of higher-order structures such as peptides from amino acids by mechanical forces had mostly only been hypothesized, ${ }^{49}$ and mechanochemical oligomerization of amino acids has been predicted to require high compressive loads and shear rates. ${ }^{50}$ In this work, we show that the mechanical activation achieved by ball milling is enough to induce peptide bond formation in a sample of unactivated glycine in the absence of bulk liquids. The oligomerization of glycine into linear oligomers Gly $\geq 2$ improves in the presence of prebiotically plausible additives such as $\mathrm{TiO}_{2},{ }^{51,52}$ and even proceeds in the presence of water. The milling temperature is shown to be critical for the oligomerization of glycine, enabling a maximum calculated total yield of oligomers Gly $\geq 2$ of $10.2 \%$ at $100{ }^{\circ} \mathrm{C}$, and peptides as long as Gly 14 were detected by a here-developed UPLC-MS method. Experiments using DKP, Gly 2 , or $\mathrm{Gly}_{3}$ as starting materials demonstrated that the mechanochemical peptide bond formation is a dynamic and reversible process with simultaneous forming and breaking of peptide bonds. Importantly, DKP, which is often regarded as a dead-end for the prebiotic formation of peptides, is a productive reactant for peptides under mechanochemical conditions. The findings of this study provide an alternative synthetic approach towards oligopeptides that not only complements the well-established alternating hot/cool prebiotic peptide bond formation, ${ }^{16-19}$ but since our protocol is not limited by poor diffusion of the solid reactants, the mechanochemical peptide bond formation circumvents the need for fluctuating dry/wet environments. 


\section{References}

1. Pinter-Frenkel, M., Samanta, M., Ashkenasy, G. and Leman, L. J. Prebiotic Peptides: Molecular Hubs in the Origin of Life. Chem. Rev. https://doi.org/10.1021/acs.chemrev.9b00664 (2020)

2. Danger, G., Plasson, R. and Pascal, R. Pathways for the formation and evolution of peptides in prebiotic environments. Chem. Soc. Rev. 41, 5416-5429 (2012).

3. Rode, B. M. Peptides and the origin of life. Peptides 20, 773-786 (1999).

4. Weber, A. L. and Pizzarello, S. The peptide-catalyzed stereospecific synthesis of tetroses: a possible model for prebiotic molecular evolution. Proc. Natl Acad. Sci. USA 103, 12713-12717 (2006).

5. Tagami, S., Attwater, J. and Holliger, P. Simple peptides derived from the ribosomal core potentiate RNA polymerase ribozyme function. Nat. Chem. 9, 325-322 (2017).

6. Chatterjee, S. A symbiotic view of the origin of life at hydrothermal impact crater-lakes. Phys.Chem.Chem.Phys. 18, 20033-20046 (2016).

7. Saad, N. Y. A ribonucleopeptide world at the origin of life. J. Syst. Evol. 56, 1-13 (2018).

8. Krishnamurthy, R. Giving rise to Life: Transition from prebiotic chemistry to protobiology. Acc. Chem. Res. 50, 455-459 (2017).

9. Miller, S. L. A Production of amino acids under possible primitive earth conditions. Science 117, 528-529 (1953).

10. Fox, S. and Harada, K. Thermal copolymerization of amino acids to a product resembling protein. Science 128, 1214 (1958). 
11. Fox, S. W. and Harada, K. The thermal copolymerization of amino acids common to protein. J. Am. Chem. Soc. 82, 3745-3751 (1960).

12. Fox, S. Thermal polymerizations of amino-acids and production of formed microparticles on lava. Nature 201, 336-337 (1964).

13. Wang, X. Challenges and outlook for catalytic direct amidation reactions. Nat. Catal. 2, 98-102 (2019).

14. Martin, R. B. Free energies and equilibria of peptide bond hydrolysis and formation. Biopolymers 45, 351-353 (1998).

15. Koshland, D. E. Kinetics of peptide bond formation. J. Am. Chem. Soc. 73, 4103-4108 (1951).

16. Lahav, N., White, D. and Chang, S. Peptide formation in the prebiotic era: thermal condensation of glycine in fluctuating clay environments. Science 201, 67-69 (1978).

17. Forsythe, J. G., Yu, S.-S., Mamajanov, I., Grover, M. A., Krishnamurthy, R., Fernandez, F. M. and Hud, N. V. Ester-Mediated Amide Bond Formation Driven by Wet-Dry Cycles: A Possible Path to Polypeptides on the Prebiotic Earth. Angew. Chem. Int. Ed. 54, $9871-9875(2015)$.

18. Rodriguez-Garcia, M., Surman, A. J., Copper, G. J. T., Suárez-Marina, I., Hosni, Z., Lee, M. P. and Cronin, L. Formation of oligopeptides in high yield under simple programmable conditions. Nat. Commun. 6, 8385, (2015).

19. Campbell, T. D., Febrian, R., McCarthy, J. T., Kleinschmidt, H. E., Forsythe, J. G. and Bracher, P. J. Prebiotic condensation through wet-dry cycling regulated by deliquescence. Nat. Commun. 10, 4508, (2019). 
20. Deamer, D. and Ross, D. S. Dry/wet cycling and the thermodynamics and kinetics of prebiotic polymer synthesis. Life 6, 28 (2016).

21. Petrus, J. A., Ames, D. E. and Kamber, B. S. On the track of the elusive Sudbury impact: geochemical evidence for a chondrite or comet bolide. Terra Nova 27, 9-20 (2015).

22. Pizzarello, S. The chemistry of Life's origin: A carbonaceous meteorite perspective. Acc. Chem. Res. 39, 231-237 (2006).

23. Burton, A. S., Stern, J. C., Elsila, J. E., Glavin, D. P. and Dworkin, J. P. Understanding prebiotic chemistry through the analysis of extraterrestrial amino acids and nucleobases in meteorites. Chem. Soc. Rev. 41, 5459-5472 (2012).

24. Lange, J., Djago, F., Eddhif, B., Blancart Remaury, Q., Ruf, A., Karpel Vel Leitner, N., Le Sergeant d'Hendecourt, L., Danger, G., Geffroy Rodier, C., Papot, S. and Poinot, P. Evidence for Proteogenic Peptide-like Sequences in Meteorites Through an EnzymeCatalysed Stereoselective Hydrolysis Strategy. ChemRxiv DOI: 10.26434/chemrxiv.11855856.v1 (2020).

25. Hansma, H. G. Mechanical energy before chemical energy at the origins of Life? Sci $\mathbf{1}$, 50 (2019).

26. Pill, M. F., East, A. L. L., Marx, D., Beyer, M. K. and Clausen-Schaumann, H. Mechanical activation drastically accelerates amide bond hydrolysis, matching enzyme activity. Angew. Chem. Int. Ed. 58, 9787-9790 (2019).

27. James, S. L. et al. Mechanochemistry: opportunities for new and cleaner synthesis. Chem. Soc. Rev. 41, 413-447 (2012).

28. Friščić, T., Mottillo, C. and Titi, H. M. Mechanochemistry for synthesis. Angew. Chem. Int. Ed. 59, 1018-1029 (2020) 
29. Howard, J. L., Cao, Q. and Browne, D. L. Mechanochemistry as an emerging tool for molecular synthesis: what can it offer? Chem. Sci. 9, 3080-3094 (2018).

30. Hernández, J. G. and Bolm, C. Altering product selectivity by mechanochemistry. $J$. Org. Chem. 82, 4007-4019 (2017).

31. Bonnamour, J., Métro, T.-X., Martinez, J. and Lamaty, F. Environmentally benign peptide synthesis using liquid-assisted ball-milling: application to the synthesis of Leuenkephalin. Green Chem. 15, 1116-1120 (2013).

32. Bolm, C. and Hernández, J. G. From synthesis of amino acids and peptides to enzymatic catalysis: A bottom-up approach in mechanochemistry. ChemSusChem, 11, 1410-1420 (2018).

33. Hernández, J. G., Frings, M. and Bolm, C. Mechanochemical enzymatic kinetic resolution of secondary alcohols under ball-milling conditions. ChemCatChem $\mathbf{8}, 1769$ 1772 (2016).

34. Ardila-Fierro, K. J., Crawford, D. E., Körner, A., James, S. L., Bolm, C. and Hernández, J. G. Papain-catalysed mechanochemical synthesis of oligopeptides by milling and twin-screw extrusion: application in the Juliá-Colonna enantioselective epoxidation. Green Chem. 20, 1262-1269 (2018).

35. Kaabel, S., Friščić, T. and Auclair, K. Mechanoenzymatic transformations in the absence of bulk water: A More Natural Way of Using Enzymes. ChemBioChem 21, $742-758(2020)$

36. Bolm, C., Mocci, R., Schumacher, C., Turberg, M., Puccetti, F. and Hernández, J. G. Mechanochemical activation of iron cyano complexes: A prebiotic impact scenario for the synthesis of $\alpha$-amino acid derivatives. Angew. Chem. Int. Ed. 57, 2423-2426 (2018). 
37. Hernández, J. G., Turberg, M., Schiffers, I. and Bolm, C. Mechanochemical Strecker Reaction: Access to $\alpha$-Aminonitriles and Tetrahydroisoquinolines under Ball-Milling Conditions. Chem. Eur. J. 22, 14513-14517 (2016).

38. Lamour, S., Pallman, S., Hass, M. and Trapp, O. Prebiotic sugar formation under nonaqueous conditions and mechanochemical acceleration. Life 9, 52 (2019).

39. Hass, M., Lamour, S., Christ, S. B. and Trapp, O. Mineral-mediated carbohydrate synthesis by mechanical forces in a primordial geochemical setting. Commun Chem $\mathbf{3}$, 140 (2020).

40. Eguaogie, O., Vyle, J. S., Conlon, P. F., Gîlea, M. A. and Liang, Y., Mechanochemistry of nucleosides, nucleotides and related materials. Beilstein J. Org. Chem. 14, 955-970 (2018).

41. Thorpe, J., O’Reilly, D., Friščić, T. and Damha, M. J. Mechanochemical synthesis of short DNA fragments. Chem. Eur. J. 26, 8857-8861 (2020).

42. Stolar, T., Lukin, S., Rajić Linarić, M., Etter, M., Užarević, K., Meštrović, E. and Halasz, I. DNA-specific selectivity in pairing of model nucleobases in the solid state. Chem. Commun. DOI: 10.1039/D0CC03491F (2020).

43. Campbell, T. D., Febrian, R., Kleinschmidt, H. E., Smith, K. A. and Bracher, P. J. Quantitative Analysis of Glycine Oligomerization by Ion-Pair Chromatography. ACS Omega 4, 12745-12752, (2019).

44. Nagayama, M., Takaoka, O., Inomata, K. \& Yamagata, Y. Diketopiperazine-mediated peptide formation in aqueous solution. Orig Life Evol Biosph 20, 249-257, (1990). 
45. Cindro, N., Tireli, M., Karadeniz, B., Mrla, T. and Užarević, K. Investigations of Thermally Controlled Mechanochemical Milling Reactions. ACS Sustainable Chem. Eng. 7, 16301-16309, (2019).

46. Lambert, J. F. Adsorption and Polymerization of Amino Acids on Mineral Surfaces: A Review. Orig Life Evol Biosph 38, 211-242, (2008).

47. Silverstein, R. M., Webster, F. X., Kiemle D. J. Spectrometric identification of organic compounds., 7th edition, John Wiley \& Sons, Inc., 2005.

48. Rimola, A., Fabbiani, M., Sodupe, M., Ugliengo, P. and Martra, G. How Does Silica Catalyze the Amide Bond Formation under Dry Conditions? Role of Specific Surface Silanol Pairs. ACS Catal. 8, 4558-4568, (2018).

49. Hansma, H. G. Mechanical energy before chemical energy at the origins of Life? Sci 1, 50 (2019).

50. Steele, B. A., Goldman, N., Kuo, I-F. W. and Kroonblawd, M. P. Mechanochemical synthesis of glycine oligomers in a virtual rotational diamond anvil cell. Chem. Sci. 11, 7760, (2020).

51. Martra, G., Deiana, C., Sakhno, Y., Barberis, I., Fabbiani, M., Pazzi, M. and Vincenti, M. The Formation and Self-Assembly of Long Prebiotic Oligomers Produced by the Condensation of Unactivated Amino Acids on Oxide Surfaces. Angew. Chem. Int. Ed. 53, 4671-4674, (2014).

52. Pantaleone, S., Ugliengo, P., Sodupe, M. and Rimola, A. When the Surface Matters: Prebiotic Peptide-Bond Formation on the $\mathrm{TiO}_{2}$ (101) Anatase Surface through Periodic DFT-D2 Simulations. Chem. Eur. J. 24, 16292-16301, (2018). 